\title{
Original
}

\section{Transplantation of mature adipocyte-derived dedifferentiated fat cells into three-wall defects in the rat periodontium induces tissue regeneration}

\author{
Daigo Suzuki1), Daisuke Akita2), Niina Tsurumachi3), Koichiro Kano ${ }^{4}$, \\ Katsuyuki Yamanaka5), Tadashi Kaneko5), Eisuke Kawano1), Shinya Iguchi1), \\ Taku Toriumi6,7), Yoshinori Arai ${ }^{8)}$, Taro Matsumoto9), Shuichi Sato 10,11), \\ and Masaki Honda $\left.{ }^{12}\right)$
}

1)Division of Applied Oral Sciences, Nihon University Graduate School of Dentistry, Tokyo, Japan

2)Department of Partial Denture Prosthodontics, Nihon University School of Dentistry, Tokyo, Japan

3)Department of Orthodontics, Nihon University School of Dentistry, Tokyo, Japan

4)Laboratory of Cell and Tissue Biology, College of Bioresource Sciences, Nihon University, Fujisawa, Japan ${ }^{5) G C ~ C o r p ., ~ T o k y o, ~ J a p a n ~}$

6)Department of Anatomy, Nihon University School of Dentistry, Tokyo, Japan

7)Division of Functional Morphology, Dental Research Center, Nihon University School of Dentistry,

Tokyo, Japan

${ }^{8)}$ Nihon University School of Dentistry, Tokyo, Japan

${ }^{9}$ Department of Functional Morphology, Division of Cell Regeneration and Transplantation,

Nihon University School of Medicine, Tokyo, Japan

${ }^{10)}$ Department of Periodontology, Nihon University School of Dentistry, Tokyo, Japan

11)Division of Advanced Dental Treatment, Dental Research Center, Nihon University School of Dentistry, Tokyo, Japan

${ }^{12)}$ Department of Oral Anatomy, Aichi Gakuin University School of Dentistry, Nagoya, Japan

(Received December 22, 2016; Accepted January 11, 2017)

\begin{abstract}
The transplantation of dedifferentiated fat (DFAT) cells in combination with poly $(d, l$-lacticco-glycolic acid) (PLGA) scaffolds has previously been proven as an effective approach in promoting periodontal tissue regeneration in a rat fenestration defect model. The aim of this study was to assess the regenerative potential of DFAT cells in a rat model of three-wall periodontal bone defect. Three-wall bone defects were created bilaterally on the mesial side of rat maxillary first molars and were either left untreated
\end{abstract}

Correspondence to Dr. Masaki Honda, Department of Oral Anatomy, Aichi Gakuin University School of Dentistry, 1-100 Kusumoto-cho, Chikusa-ku, Nagoya, Aichi 464-8650, Japan Fax:+81-52-752-5988 E-mail: honda-m@dpc.agu.ac.jp

Color figures can be viewed in the online issue at J-STAGE. doi.org/10.2334/josnusd.16-0878

DN/JST.JSTAGE/josnusd/16-0878 or treated by implantation of PLGA scaffolds with DFAT cells or PLGA alone. Four weeks after surgery, the tissues were processed for micro-computed tomography (micro-CT) and histomorphometric examination. Micro-CT revealed that the PLGA/ DFAT group had significantly higher rates of bone regeneration than the other groups, while histomorphometric analysis showed that the PLGA/DFAT group had significantly higher densities of collagen fiber bundles in acellular and cellular cementum than the PLGA group. Moreover, the results indicate that the placement of the PLGA scaffold prevented the downgrowth of the junctional epithelium. These findings suggest that DFAT cells contribute to tissue regeneration in three-wall periodontal defects, while PLGA provides space necessary for periodontal tissue restoration. 
Keywords: periodontal tissue restoration; DFAT cell; PLGA.

\section{Introduction}

Periodontitis, the most common inflammatory disease in humans, causes the destruction of connective tissue between the root of a tooth and the surrounding alveolar bone. Damaged periodontal tissues have limited potential for regeneration because of the lack of progenitor/stem cells needed to produce multiple specialized cell types within the periodontal wound (1-3). Appropriate cells, biological signals, scaffolds, blood supply, mechanical loading, and microbial control are essential for creating optimal conditions for successful periodontal tissue reconstruction $(4,5)$. Several studies have already described the application of cell-based tissue engineering for periodontal tissue regeneration (6-9). The main principal of tissue engineering is to expand certain types of tissue-derived cells seeded on a biodegradable scaffold, which is subsequently implanted into a wounded/defective site for restoration of single or multi-component tissues, such as the periodontium. In dentistry, bone marrow mesenchymal stromal cells and periodontal ligament-derived stem cells are widely used for periodontal tissue regeneration $(7,8,10)$. Adipose-derived stem cells (ASCs) are another promising cell type for periodontal tissue engineering considering that adipose tissue containing a considerable number of stromal cells can be obtained through a less invasive method and in larger quantities than bone marrow (11). The utility of ASCs for the restoration of cementum, alveolar bone, and periodontal ligament structures has been demonstrated in rat and canine models, suggesting that ASCs potentially contribute to periodontal tissue regeneration (12-14). However, the optimal stem cell type for periodontal tissue engineering has not yet been established, while findings remain controversial.

Adipose tissue is composed mostly of mature adipocytes containing large single lipid droplets that have lost their proliferative ability. A recent study using a ceiling culture technique based on adipocyte buoyancy showed that adipocytes could be converted into fibroblast-like cells known as dedifferentiated fat (DFAT) cells (15). DFAT cells can re-establish their active proliferation ability and differentiate into various cell types under appropriate cell culture conditions. Both ASCs and DFAT cells can be obtained from the buccal fat pad (BFP) located between the masseter and buccinator muscles and the ascending mandibular ramus and zygomatic arch in the oral cavity. The BFP, which is often removed during oral and maxillofacial surgery, is easily accessible using a simple surgical procedure performed by dentists and maxillofacial surgeons. We had previously isolated mature adipocytes from the BFP of five donors subjected to Le Fort I osteotomy in combination with sagittal split ramus osteotomy (SSRO) and successfully generated DFAT cells (16). Interestingly, adipocytes from all patients differentiated into DFAT cells with similar efficiency irrespective of donor age, suggesting that even the oral cavity of mature patients can provide suitable precursors for DFAT cells to be used in tissue engineering.

DFAT cells can provide several advantages over ASCs derived from the same adipose tissue. Firstly, DFAT cells can be obtained as a pure cell population, because they are produced from ceiling cultures of fat cells floating in the upper layer after centrifugation (15). In contrast, ASCs are acquired from the stromal vascular fraction accumulating at the bottom layer (pellet), which includes other cell types besides ASCs. Therefore, further isolation steps are required to obtain high-purity ASC cultures. Secondly, many more DFAT cells (up to five times) can be generated from a given amount of fat tissue compared to ASCs (15). Furthermore, according to our previous study, DFAT cells have higher osteoblastic and adipogenic differentiation capacity than ASCs in vitro (12). Finally, DFAT cells express genes involved in the regulation of differentiation and tissue-specific functions, such as SFRP2, PRRX1, HEY2, AEBP1, PEG10, PRRX2, $R U N X 1, F Z D 7$, and IGFBP5 (17), indicating their potential as progenitors for various cell lineages. These results suggest that DFAT cells are a more promising cell type for tissue regeneration compared to ASCs.

We had previously demonstrated the effectiveness of a poly $(d, l$-lactic-co-glycolic acid) (PLGA) scaffold in the correction of fenestration bone defects created in mandibular alveolar bone in vivo $(12,13)$. PLGA, a biodegradable polymer with excellent biocompatibility, has a higher structural integrity compared to soft scaffolds made from hydrogels and collagen sponge $(7,18-23)$. Transplantation of a PLGA scaffold into the fenestration cavity allowed the space to become filled with connective tissue, while no inflammation was observed during the 5 -week experimental period. Furthermore, new connective tissue was formed around the remaining scaffold (12). These results suggest that the PLGA scaffold contributes to the preservation of space for tissue regeneration within the periodontium. Moreover, the transplantation of PLGA scaffolds carrying DFAT cells promoted cementum and alveolar bone formation, demonstrating higher potential for healing of fenestration bone defects in a rat model 


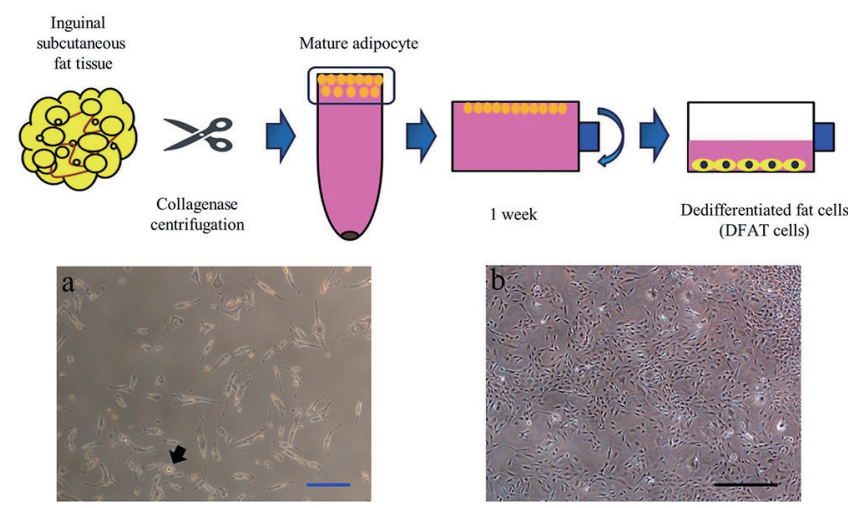

Fig. 1 Generation of rat DFAT cells. The upper panel illustrates the method used to isolate DFAT cells from floating rat unilocular adipocytes. Floating cells attached to the upper surface of the flasks produced DFAT cells by asymmetrical division for 1 week. (a) Cells containing small lipid droplets (arrow); blue scale bar, $100 \mu \mathrm{m}$. (b) Flasks that were inverted for 1 week formed a layer of DFAT cells at the bottom of the culture dish. Black scale bar, $500 \mu \mathrm{m}$.

\section{(12).}

Overall, these data indicate that DFAT cells are a promising cell type used for periodontal tissue regeneration. However, it remains unclear whether DFAT cells could promote the reduction of the downgrowth of the junctional epithelium and enhance the formation of the alveolar bone in three-wall defects. The aim of the present study was to evaluate the potential of DFAT cells seeded on a PLGA scaffold to regenerate periodontal tissue using a rat model of three-wall defects created in the mandibular alveolar bone.

\section{Materials and Methods}

\section{Experimental animals}

All animal experiments were reviewed and approved by the Animal Experimentation Committee of Nihon University School of Dentistry (AP15D023).

\section{Isolation of rat DFAT cells}

DFAT cells were isolated from mature adipocytes of an 8-week-old male F344 rat (body weight $190 \pm 10 \mathrm{~g}$; CLEA Japan Inc., Tokyo, Japan) using a modified method described previously (15). Briefly, approximately $1 \mathrm{~g}$ of subcutaneous fat tissue was washed extensively with phosphate-buffered saline (PBS; Wako, Osaka, Japan), minced, and digested in $0.1 \%(\mathrm{w} / \mathrm{v})$ collagenase solution (Sigma-Aldrich, St. Louis, MO, USA) at $37^{\circ} \mathrm{C}$ for 60 min. After filtration and centrifugation at $135 \mathrm{~g}$ for $3 \mathrm{~min}$, cells floating at the top layer were collected, and $5 \times 10^{4}$ cells were placed in $12.5-\mathrm{cm}^{2}$ culture flasks (BD Falcon, Corning, NY, USA) filled up to the top with Dulbecco's modified Eagle's medium (DMEM; Sigma Aldrich Japan,

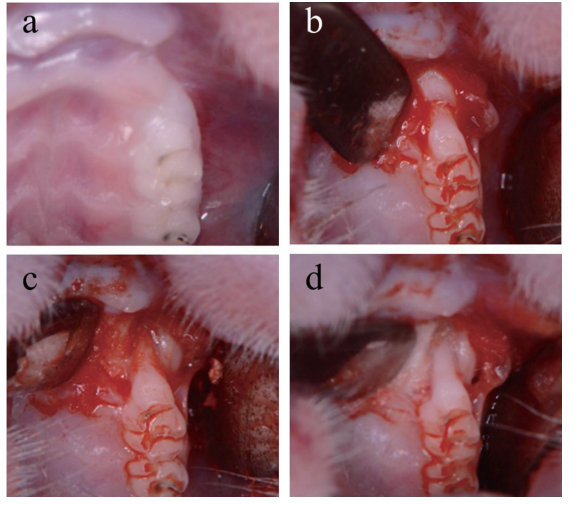

Fig. 2 Surgical creation of a three-wall alveolar bone concavity at the mesial root surface of maxillary first molars. (a) Overview of the surgical site at the maxillary right first molar. (b) A 3-mm full thickness incision was made to expose the mesial root surface and alveolar bone. (c) A three-wall defect was created using a piezoelectric device. (d) Acellular and cellular cementum was completely removed using a hand scaler.

Osaka, Japan) supplemented with $20 \%$ fetal bovine serum (FBS; Nichirei Bioscience Inc., Tokyo, Japan) and incubated at $37^{\circ} \mathrm{C}$ in $5 \% \mathrm{CO}_{2}$. Mature adipocytes floated up and adhered to the top inner surface of the culture flasks. After 1 week, $80 \%$ of the medium was removed, and the flasks were inverted so that the cells would settle at the bottom (Fig. 1). The medium was changed every 4 days. Cuboidal cells formed colonies on day 7 (Fig. 1a) as described in our previous study (12). Cells reaching $80 \%$ confluence were split, and second-passage DFAT cells were used for experiments (Fig. 1b).

\section{Preparation of the cell-scaffold complex}

Solid PLGA scaffolds (lactic acid:glycolic acid = 75:25, MW 25 kDa; GC Corporation, Tokyo, Japan) with a porosity of $80-90 \%$, were prepared as previously described $(12,13,22)$. Briefly, PLGA scaffolds were resized to approximately $2 \times 2 \times 2 \mathrm{~mm}$, soaked in $70 \%$ ethanol for $30 \mathrm{~min}$, and then washed three times with PBS to remove the residual ethanol. Thereafter, $100-\mu \mathrm{L}$ aliquots of cell suspension $\left(1 \times 10^{7} \mathrm{cells} / \mathrm{mL}\right)$ were seeded on top of pre-wetted solid PLGA scaffolds and left undisturbed in an incubator for $1 \mathrm{~h}$. Empty solid PLGA scaffolds were used as control.

\section{In vivo experiments and surgical procedure}

A total of 20 healthy male F344 rats (8 weeks old, 200 $\pm 10 \mathrm{~g}$ ) were used for in vivo experiments. The animals were pre-anesthetized by isoflurane inhalation and then subjected to general anesthesia by intraperitoneal injection of a mixture of $0.15 \mathrm{mg} / \mathrm{kg}$ dexmedetomidine hydrochloride, $2.0 \mathrm{mg} / \mathrm{kg}$ midazolam, and $2.5 \mathrm{mg} /$ 
Table 1 Distance from the cement-enamel junction to the alveolar bone crest on micro-CT (mm)

\begin{tabular}{llllc}
\hline Time after surgery & no-surgery & no-transplant & PLGA & PLGA/DFAT \\
\hline 1 week & $0.15 \pm 0.10$ & $1.98 \pm 0.04$ & $1.96 \pm 0.04$ & $1.92 \pm 0.08$ \\
3 weeks & $0.15 \pm 0.10$ & $1.48 \pm 0.14^{*}$ & $1.28 \pm 0.18^{*}$ & $0.78 \pm 0.22$ \\
4 weeks & $0.14 \pm 0.10$ & $1.19 \pm 0.18^{* *}$ & $0.95 \pm 0.20^{*}$ & $0.16 \pm 0.12$ \\
\hline$* P<0.05, * *<0.01$ compared to the PLGA/DFAT group by one-way ANOVA $(n=5)$. &
\end{tabular}

Table 2 Histomorphometric analysis at 4 weeks after surgery expressed as area (mm)

\begin{tabular}{|c|c|c|c|c|}
\hline & no-surgery & no-transplant & PLGA & PLGA/DFAT \\
\hline Cement-enamel junction to alveolar bone crest & $0.62 \pm 0.10$ & $1.44 \pm 0.51 * *$ & $1.25 \pm 0.45^{*}$ & $0.65 \pm 0.25$ \\
\hline Relative epithelial downgrowth & $0.42 \pm 0.18$ & $1.23 \pm 0.82 * *$ & $0.44 \pm 0.31$ & $0.42 \pm 0.22$ \\
\hline Relative periodontal ligament width (cellular cementum side) & $0.21 \pm 0.03$ & $0.20 \pm 0.05$ & $0.22 \pm 0.04$ & $0.18 \pm 0.02$ \\
\hline Relative periodontal ligament width (acellular cementum side) & $0.22 \pm 0.06$ & $0.23 \pm 0.04$ & $0.24 \pm 0.08$ & $0.21 \pm 0.02$ \\
\hline
\end{tabular}

$* P<0.05,{ }^{*} P<0.01$ compared to the PLGA/DFAT group by one-way ANOVA $(n=5)$.

$\mathrm{kg}$ butorphanol tartrate. Lidocaine $(0.5 \mathrm{~mL}, 1: 80,000$ dilution, Xylocaine; Astra Zeneca, Osaka, Japan) was administered by intraperitoneal injection to control bleeding and provide additional anesthesia. A 3-mm full thickness incision was made along the alveolar ridge mesial to the maxillary first molar (M1). Flaps were elevated to expose the root surface and surrounding alveolar bone, and three-wall bone defects were created using a piezoelectric device (K-scalar $\alpha$; Woodpeker, Inc., Guangxi, China) $(9,24,25)$. Thereafter, the residual bone, periodontal ligament, and cementum of the M1 root were carefully removed using a hand scaler. The size and contour of the defect $(2 \times 2 \times 2 \mathrm{~mm}$, length $\times$ width $\times$ depth) were finalized by constantly monitoring the dimensions using a clinical periodontal probe. Afterwards, the created concavities were rinsed with sterile saline and dried with sterile gauze. Rats were then randomly divided into three groups ( $n=5$ per group): the PLGA/DFAT group received DFAT cell-loaded PLGA scaffolds, the PLGA group received acellular scaffolds, and the negative control (no-transplant) group was left untreated. Animals not subjected to surgery (no-surgery group) were used as positive control $(n=5)$ (Fig. 2). The scaffolds were implanted immediately after surgery. Afterwards, the flaps were repositioned and sutured with resorbable sutures.

\section{In vivo $\mathrm{X}$-ray micro-computed tomography (micro-CT) imaging}

In vivo $\mathrm{X}$-ray micro-CT ( $\mathrm{R} \_\mathrm{mCT}$; Rigaku Corporation, Tokyo, Japan) was performed as described previously $(13,26)$. The exposure parameters were $17 \mathrm{~s}, 90 \mathrm{kV}$, and $100 \mu \mathrm{A}$, while the isotropic voxel size was $30 \mu \mathrm{m}$.

Hard-tissue images were obtained from individual rats immediately after surgery ( 0 week) and every week for 4 weeks thereafter, while three-dimensional images were constructed using i-View (J. Morita Co., Kyoto, Japan).
Bone volume within the region of interest (ROI) was measured from voxel images using the bone volumemeasuring software 3 by 4 viewer 2011 (Kitasenjyu Radist Dental Clinic, I-View Image Center, Tokyo, Japan). The ROI size was $2 \times 2 \times 2 \mathrm{~mm}$, which covered the surgical defect. To assess re-ossification of the created concavity, the bone volume in the ROI and the distance from the cement-enamel junction (CEJ) to the alveolar bone crest (ABC) were measured (Table $1, n=5$ per group).

\section{Histological analysis}

Four weeks after surgery, rats were euthanized by excess $\mathrm{CO}_{2}$ gas inhalation, and the harvested tissue specimens were fixed in $10 \%$ neutral buffered paraformaldehyde for $24 \mathrm{~h}$, decalcified in $10 \%$ ethylenediaminetetraacetic acid (EDTA) for 5 weeks, dehydrated through a graded series of ethanol solutions, and embedded in paraffin. Frontal plane sections ( $4 \mu \mathrm{m}$ thick) were prepared using a Leica RM2165 microtome (Leica, Nussloch, Germany) and stained using hematoxylin and eosin (HE) for general tissue evaluation. To observe principal and Sharpey's fibers in the periodontal ligament and lamina propria, the sections were subjected to Azan staining.

Osteoclasts and odontoclasts were defined as multinucleated ( $\geq 3$ ) tartrate-resistant acid phosphatase (TRAP)-positive cells in contact with the surface of the alveolar bone and cellular cement, respectively. Four weeks after surgery, TRAP-positive cells were detected by TRAP staining performed using a leukocyte acid phosphatase kit (Sigma-Aldrich) (6). Images were captured under a microscope (Eclipse LV100POL; Nikon, Tokyo, Japan), and TRAP-positive cells were counted using the Image J software (NIH, Bethesda, MD, USA).

\section{Histomorphometric analysis}

For quantitative analysis, three sections per staining method in each specimen were evaluated under a light 


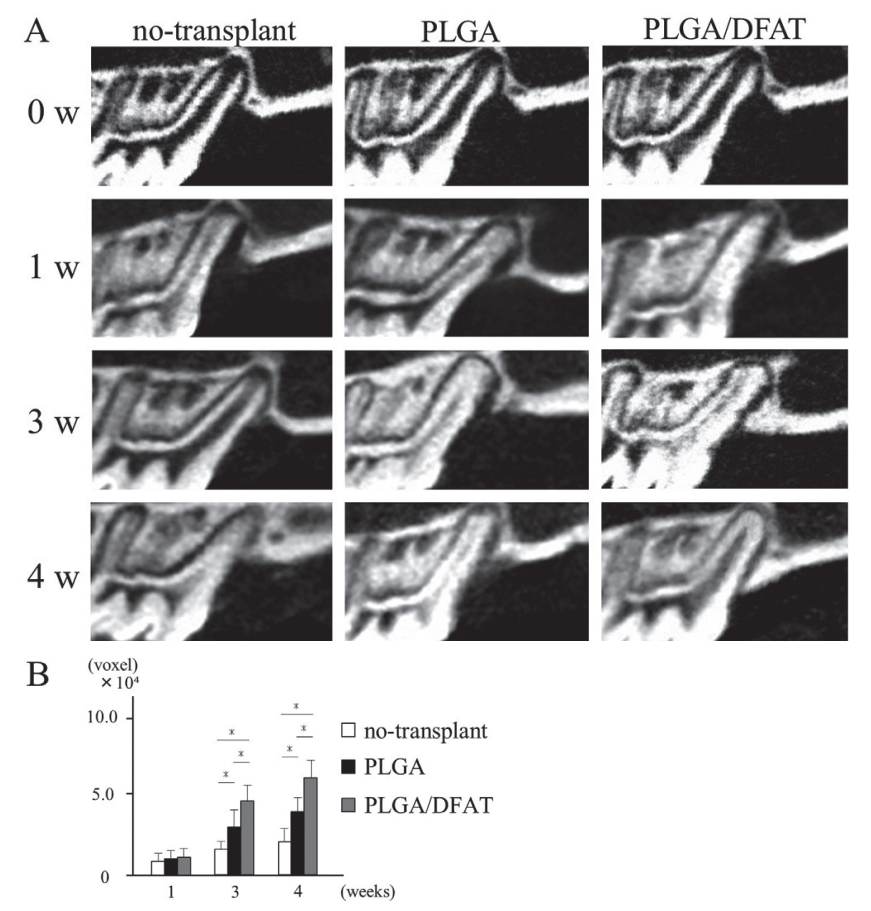

Fig. 3 New bone formation in the three-wall alveolar bone concavity. (A) Two-dimensional micro-CT images of rat maxillary right first molars at $0,1,3$, and 4 weeks after surgery in the no-implant, PLGA, and PLGA/DFAT groups. Three-dimensional images were reconstructed using i-View. (B) Quantification of newly formed bone volume based on micro-CT images using 3-D structure analysis software. A significant difference was observed between the PLGA/DFAT and PLGA groups at weeks 3 and 4. Data are presented as mean $\pm \mathrm{SD}(n=5) ; * P<0.05$ compared to the no-implant group.

microscope (UPM Axiophot 2; Carl Zeiss Japan, Tokyo, Japan) using histomorphometry software (Axio Vision 4.7; Carl Zeiss Japan) as described previously (27). Epithelial downgrowth was measured from the CEJ to the furthermost apex of the junctional epithelium. The relative functional ligament width was measured as the distance from the surface of cellular or acellular cementum to that of the alveolar bone (Table 2).

\section{Statistical analysis}

Statistical analyses were conducted using Prism GraphPad software version 6 (Graphpad Software San Diego, CA, USA). All data were expressed as mean \pm standard deviation, while statistical comparisons between groups were performed using one-way analysis of variance (ANOVA) followed by the Tukey-Kramer post hoc test. Statistical significance was set at $P<0.05$.
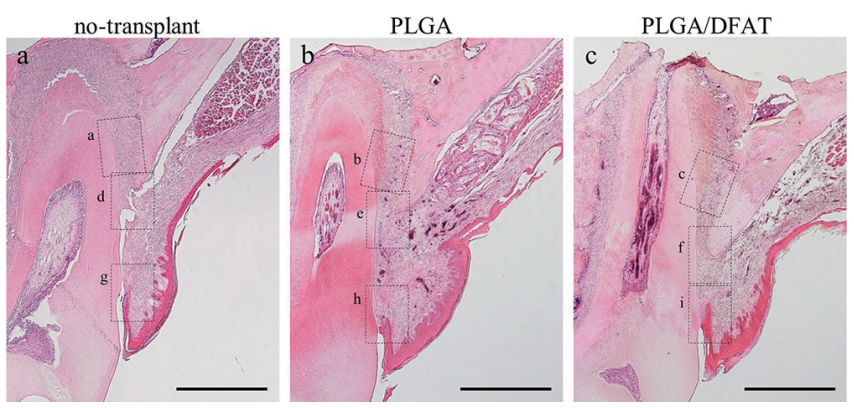

Fig. 4 Histological analysis by HE staining at 4 weeks after surgery (low-power magnification). (a) No-implant group. (b) PLGA group. (c) PLGA/DFAT group. Formation of new bone and periodontal ligament was observed in all groups. Scale bars: 1 $\mathrm{mm}$.

\section{Results}

\section{General observations}

All rats showed uneventful post-operative healing during the 4-week observation period and demonstrated similar weight gain. At the time of euthanasia, soft tissues around the surgical sites healed without detectable differences among the groups. No visible adverse reactions, including root exposure, infection, or suppuration, were observed in any animal during the experimental period.

\section{Micro-CT analysis of alveolar bone formation}

To evaluate alveolar bone regeneration, reconstituted micro-CT images and horizontal sections were prepared and analyzed up to week 4 , because this was when the crest of the newly formed bone almost achieved its original position in the PLGA/DFAT group (Fig. 3, Table 1).

Continuous micro-CT analysis showed that all groups exhibited a gradual increase in bone volume and a gradual decrease in the width of the periodontal ligament space compared to the baseline at the time of surgery (week 0) (Fig. 3A, B). No significant difference in bone volume was found among the groups at 1 and 2 weeks after surgery (Fig. 3B) (week 2 images not shown). However, after 3 and 4 weeks, the PLGA/DFAT group showed significantly greater bone volume compared to the other two groups $(P<0.05$; Fig. 3B). After 4 weeks, the PLGA/DFAT group exhibited full bone formation and a pronounced alveolar bone crest (Fig. 3A).

The distance from the CEJ to the $\mathrm{ABC}$ was measured at weeks 1-4 (Table 1). A significant difference in the CEJ-ABC distance was observed between the PLGA/ DFAT and PLGA groups at 3 and 4 weeks after surgery $(P<0.05)$. However, no significant difference in the CEJ-ABC distance was observed between the PLGA/ DFAT group $(0.16 \pm 0.12 \mathrm{~mm})$ and the no-surgery group 


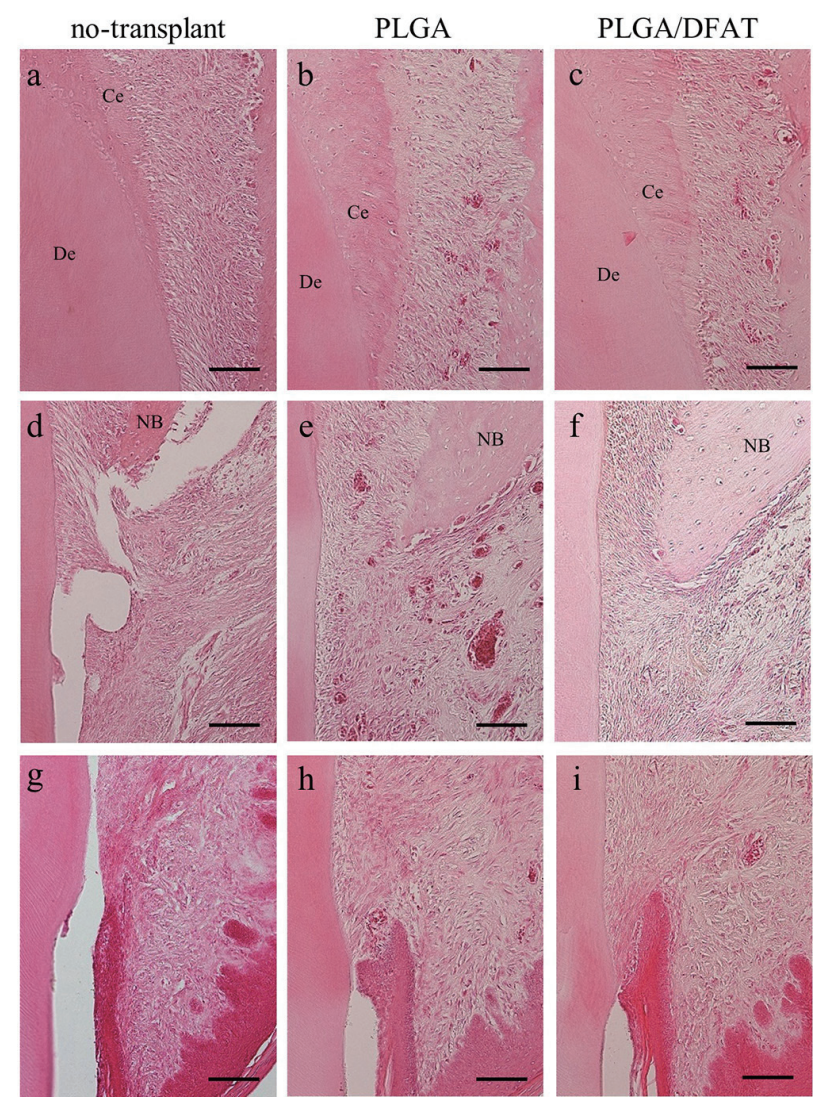

Fig. 5 Histological analysis by HE staining at 4 weeks after surgery (medium-power magnification). Areas framed in Fig. 4 are magnified. (a, d, g) No-implant group. (b, e, h) PLGA group. $(c, f, i)$ PLGA/DFAT group. Cellular cementum and the alignment of the periodontal ligaments $(\mathrm{a}, \mathrm{b}, \mathrm{c})$. Acellular cementum, the lamina propria, and the crest of alveolar bone (d, e, f). Acellular cementum and gingival fiber bundles below the terminal point of the junctional epithelium ( $g, h, i)$. De: root dentin, Ce: newly formed cementum, and NB: newly formed bone. Scale bars: 100 $\mu \mathrm{m}$.

$(0.14 \pm 0.10 \mathrm{~mm})$, indicating that the PLGA/DFAT scaffolds were able to restore the tissues to their original state.

\section{Periodontal tissue regeneration}

We first confirmed that the original alveolar bone and periodontal ligament were completely removed from the mesial side of the maxillary M1 molar mesial root (data not shown). Both cellular and acellular cementum had also been completely removed from the M1 mesial root surface, whereas cellular cementum in the apical area of the mesial root was retained (data not shown). The newly formed alveolar bone, cellular cementum, and periodontal ligament were clearly observed in all groups at low magnification 4 weeks after surgery. During this time, no visible residues of PLGA scaffolds were observed (Fig. 4a-c). A higher degree of cellular cementogenesis in the

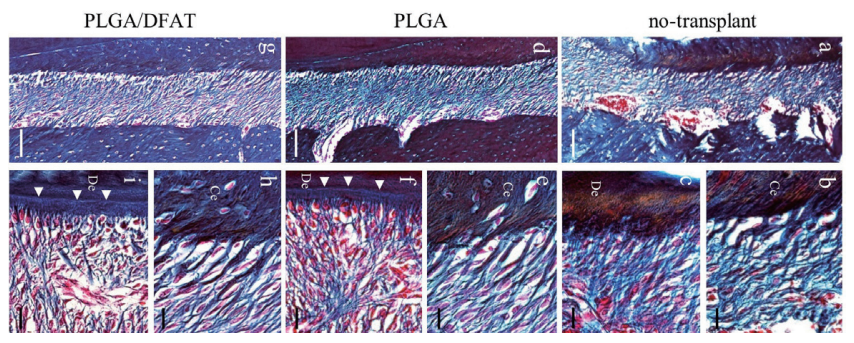

Fig. 6 Histological analysis by Azan staining at 4 weeks after surgery. (a, b, c) No-implant group. (d, e, f) PLGA group. (g, h, i) PLGA/DFAT group. Low-magnification images show the alignment of collagen fiber bundles (a, d, g). High-magnification images show the insertion of collagen fiber bundles into cellular cementum (b, e, h) and acellular cementum (c, f, i). De: root dentin, Ce: newly formed cementum, and arrowheads: acellular cementum. White scale bars: $100 \mu \mathrm{m}$; black scale bars: $10 \mu \mathrm{m}$.

mesial side was noted in the PLGA and PLGA/DFAT groups compared to the no-transplant group, although cellular cementum of the apical area was also observed in the latter group. The CEJ-ABC distance at 4 weeks after surgery was significantly shorter in the PLGA/DFAT group compared to the other groups subjected to surgery, while being very similar to that in the no-surgery group (Fig. 4, Table 2). The relative length of the junctional epithelium was also significantly shorter in the PLGA/ DFAT and PLGA groups compared to the no-transplant group $(P<0.05)$ (Fig. 4, Table 2).

Although the downgrowth of the junctional epithelium was slightly lesser in the PLGA/DFAT group compared to the PLGA group, the difference was not statistically significant as evidenced by microscopy analysis at medium-power magnification (Fig. 5h, i; Table 2). Gingival fiber bundles in the lamina propria and principal fiber bundles in the periodontal ligament were clearly observed in the root mesial area in all groups (Fig. 5a-i). Principal fiber bundles in the periodontal ligament of the PLGA and PLGA/DFAT groups had an oblique functional orientation from cellular cementum to the alveolar bone observed in the natural periodontal ligament structure as revealed by H\&E and Azan staining (Fig. 5b, c; Fig. 6). On the other hand, gingival fiber bundles were more organized in the gingiva of the PLGA/DFAT group (Fig. 5f, i) than that of the PLGA group (Fig. 5e, h). The orientation of principal and gingival fiber bundles in the no-transplant group was irregular (Fig. 5a, g; Fig. 6a-c). No significant difference in the width of the periodontal ligament space from the alveolar bone to acellular or cellular cementum was observed among the groups (Table 2). Osteoblasts lining the organic matrix and osteocytes trapped in the lacunae of the newly formed alveolar bone were detected 

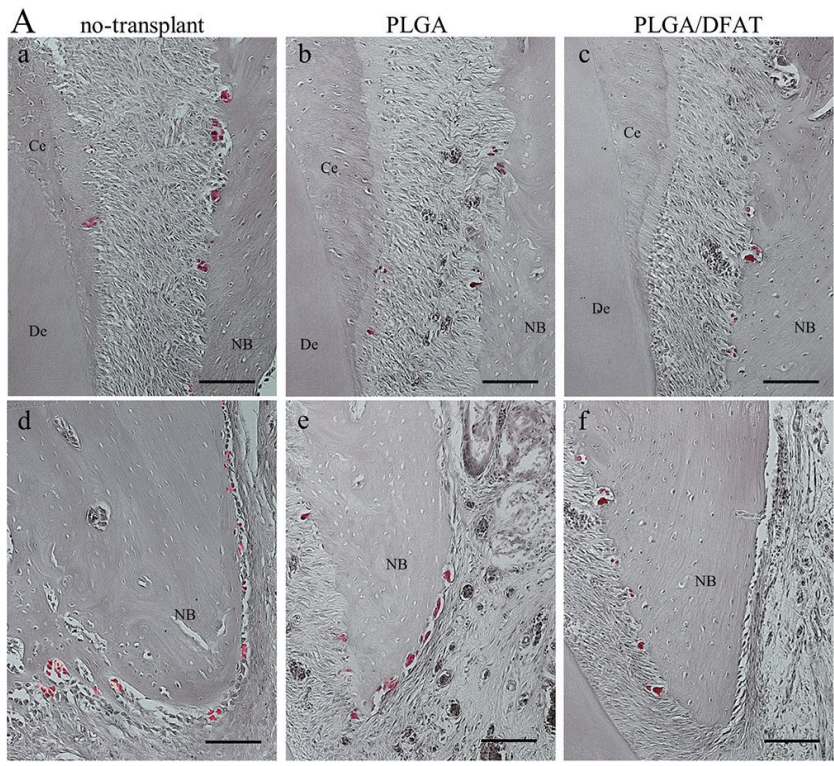

B

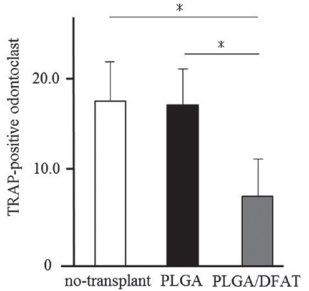

Fig. 7 Localization of TRAP-positive osteoclasts and odontoclasts. (A) Images of TRAP staining. Osteoclasts and odontoclasts localized on the alveolar bone and cellular cementum in the periodontal ligament side in the no-implant group (a) and PLGA group (b). Osteoclasts localized on the alveolar bone in the PLGA/DFAT group (c). Osteoclasts observed on the surface of the alveolar bone of the free gingiva side in the no-implant group (d), PLGA group (e), and PLGA/DFAT group (f). De: root dentin, Ce: newly formed cementum, and NB: newly formed bone (scale bars: 100 $\mu \mathrm{m})$. (B) Number of TRAP-positive cells. A significant difference was found between the PLGA/DFAT and PLGA groups. Data are presented as mean $\pm \mathrm{SD}(n=5) ; * P<0.05$.

in all groups (Figs. 5, 7). In addition, multi-nucleated TRAP-positive osteoclasts were clearly observed in the periodontal ligament side of the alveolar bone surface in all groups. A significant difference in the number of osteoclasts was observed between the PLGA and PLGA/ DFAT groups based on H\&E and TRAP staining (Figs. 5, 7). Moreover, multi-nucleated TRAP-positive cells were found at the free gingiva side of the alveolar bone surface in the no-transplant and PLGA groups but not the PLGA/ DFAT group (Fig. 7). Polymorphonuclear cells were also observed on the cellular cementum surface of the mesial root and were subsequently identified as odontoclasts by TRAP staining (Fig. 7).

At high magnification, Sharpey's fibers were clearly detected in cellular and acellular cementum of all groups
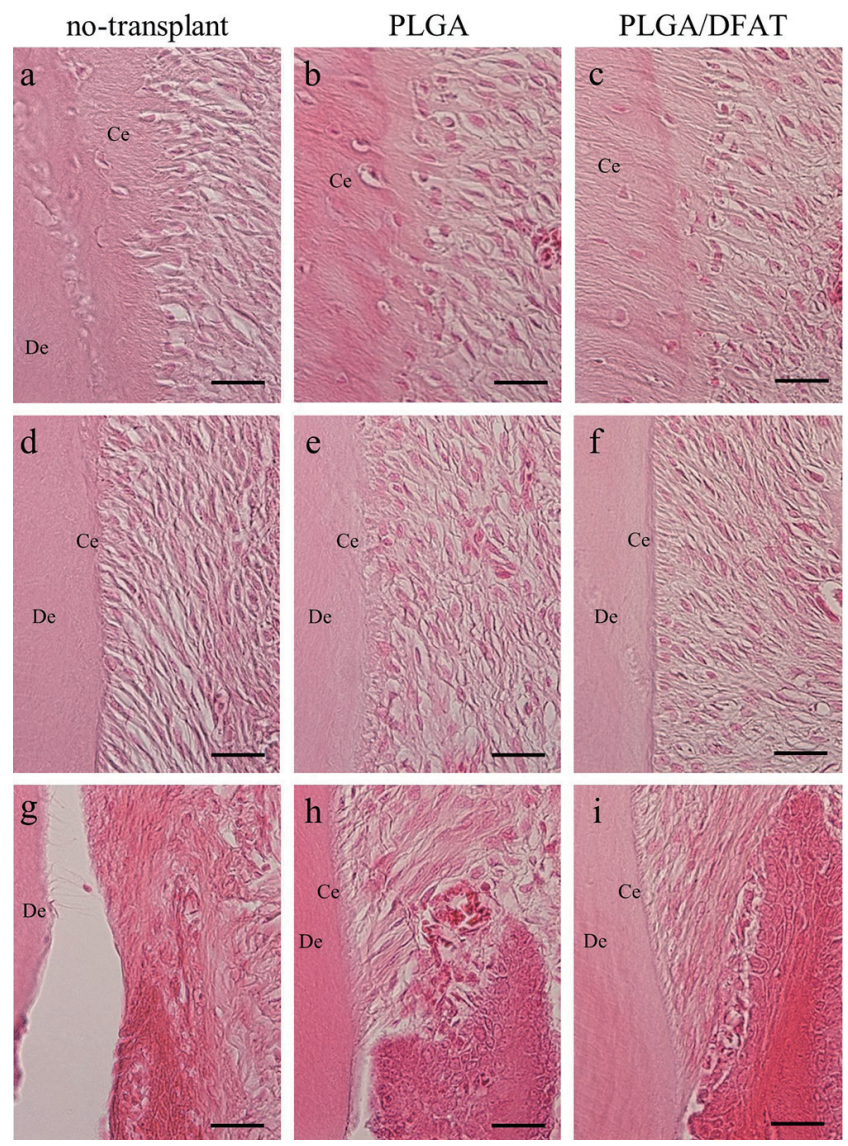

Fig. 8 Histological analysis by HE staining at 4 weeks after surgery (high-power magnification). Areas presented in Fig. 6 are magnified. (a, d, g) No-implant group. (b, e, h) PLGA group. (c, $f, i)$ PLGA/DFAT group. Insertion of principal fiber bundles in cellular cementum $(a, b, c)$. Insertion of principal fiber bundles in acellular cementum and the alignment of principal fiber bundles in the periodontal ligament (d, e, f). Insertion of the gingival fiber bundles into acellular cementum in the lamina propria below the terminal point of the junctional epithelium $(g, h, i)$. De: root dentin and Ce: newly formed cementum. Scale bars: $10 \mu \mathrm{m}$.

(Fig. 8). Principal and gingival fiber bundles positioned parallel to the root surfaces were observed in acellular cementum of all groups (Fig. 6c, f, i; Fig. 8d-f). However, principal fiber bundles were more organized in the PLGA/DFAT group compared to the PLGA group (Fig. 8e, f). Principal fiber bundles were positioned oblique or perpendicular to the root surface in all groups (Fig. 6). However, unlike the PLGA group, the PLGA/ DFAT group showed densely organized Sharpey's fibers embedded into the newly formed cellular and acellular cementum (Fig. 8b, c). The measurement of the mean distance between principle fibers (Fig. 6e, f, h, i) revealed a significant difference between the PLGA/ DFAT and PLGA groups (Table 3). Although the width of acellular cementum was similar in all groups, the 
Table 3 The measurement of the mean distance between principal fibers through histomorphometric analysis of periodontal ligament quality ( $\mathrm{mm})$

\begin{tabular}{llcc}
\hline & no-transplant & PLGA & PLGA/DFAT \\
\hline Acellular cementum side & $8.85 \pm 2.12^{*}$ & $8.65 \pm 1.84^{*}$ & $2.65 \pm 0.25$ \\
Cellular cementum side & $8.25 \pm 1.86^{*}$ & $7.25 \pm 0.69^{*}$ & $3.05 \pm 0.28$ \\
\hline${ }^{*} P<0.01$ compared to the PLGA/DFAT group by one-way ANOVA $(n=5)$. &
\end{tabular}

longitudinal length of newly formed acellular cementum was significantly shorter in the no-transplant group than in the PLGA and PLGA/DFAT groups (Fig. 8d-f).

\section{Discussion}

The objective of the current study was to test a perspective therapeutic approach to periodontal tissue regeneration based on DFAT cells seeded on PLGA scaffolds. Our previous study demonstrated that combining PLGA scaffolds with ASCs or DFAT cells was effective in promoting periodontal tissue regeneration in artificially created fenestrations of rat alveolar bone $(12,13)$. In the present study, we examined the effects of PLGA scaffolds populated with DFAT cells on the reduction of gingival recession and the formation of the junctional epithelium using a rat model of a three-wall intrabony periodontal defect. The results suggest that periodontal tissue regeneration was better with the PLGA/DFAT complex than with acellular PLGA scaffolds.

The three-wall defect model was selected for this study because it allowed us to analyze healing at the epithelial junction and connective tissue interfaces, which better resembles a clinical situation compared to our previous fenestration defect model $(12,28)$. However, intrabony defects have been argued to naturally heal better compared to fenestration (29), one-wall (30), or furcation (31) defects.

A suitable scaffold for successful tissue engineering should be biodegradable, so that it could be eventually replaced by regenerated periodontal tissue (32), and should be able to maintain a space within the wound site for appropriate tissue organization (33). The results of our study indicated that no PLGA scaffold residues could be found 4 weeks after implantation and that the regenerated tissue was similar to the original periodontium of the positive control group. Furthermore, groups implanted with PLGA scaffolds showed lesser epithelial downgrowth compared to the no-implant group, indicating that PLGA scaffolds meet the aforementioned requirements. In addition, PLGA implantation significantly stimulated the formation of new alveolar bone and acellular cementum and increased the density of gingival collagen bundles anchored into acellular cementum as extrinsic fibers, which form attachment points for connective tissue in the lamina propria just below the junctional epithelium. The PLGA scaffold placed within the intrabony concavity enabled the mucoperiosteal flap of the gingiva to return to its initial position, which did not happen in the no-implant group. The difference in flap repositioning could affect connective tissue attachment in the lamina propria, which is a key factor in restricting the migration of the junctional epithelium. Taken together, these data indicate that the PLGA scaffold could potentially preserve the wound space by preventing epithelial downgrowth.

The complementation between the PLGA scaffold and DFAT cells further improved tissue regeneration by increasing bone height as evidenced by micro-CT and histomorphometric analyses. Our previous findings indicated that DFAT cells labeled with Dil, a lipophilic membrane dye, remained in the periodontal ligament for five weeks (12) where they can secrete several cytokines associated with bone formation and angiogenesis essential for bone remodeling (34). Indeed, transplanted DFAT cells have been reported to participate in the repair of alveolar bone via secretion of trophic factors (35-38). However, no evidence has suggested that DFAT cells can differentiate into periodontal ligament cells, while further research is required to determine the mechanism underlying DFAT cell's contribution to bone regeneration.

Although the longitudinal length and width of newly formed cementum were similar in the PLGA/DFAT and PLGA groups, the number of embedded principal fiber bundles in the PLGA/DFAT group was higher based on the analysis of the interspace between fiber bundles (Table 3). These results suggest that DFAT cells contribute to the formation of principal fiber bundles rather than promote cementum deposition. The reason for this discrepancy is unclear. Nevertheless, one possible explanation is that the number of mesenchymal stem cells in the original periodontal tissue may be sufficient for the deposition of new cementum after surgery. However, the underlying mechanism remains to be determined.

The insertion of collagen fiber bundles into cementum and alveolar bone may result in improved resistance, protection, and sensing of mechanical loads from mastication (39). The addition of DFAT cells to PLGA scaffolds improved the development of the connec- 
tive tissue structure, indicating that DFAT cells were positioned over connective attachment areas. However, the organization of principal fibers in the periodontal ligament was not consistent with that of gingival fibers in the lamina propria of the PLGA group. In general, gingival connective tissue has a remarkable capacity for healing and regeneration (40). Moreover, although the connective tissue in the lamina propria had just been lifted by surgery, the original periodontal ligament was completely removed and new ligament was formed instead. Thus, we can conclude that gingival fibers were organized faster than principal fibers in the presence of DFAT cells. However, our experimental model may not entirely reflect periodontal disease in humans, given our exclusion of the effect of plaque bacteria on the emergence of three-wall defects. Therefore, whether the results obtained in this rat model translate into clinical benefits for patients remains to be clarified.

Moreover, fewer ostetoclasts and no TRAP-positive cells were observed in the PLGA/DFAT group compared to the no-implant and PLGA groups. These findings suggest that DFAT cells may either ameliorate alveolar bone resorption by blocking osteoclast recruitment or exert immunomodulatory effects by suppressing proinflammatory response through the inhibition of cytokine and chemokine secretion, the former being more likely (41). Further studies are necessary to evaluate whether the immunomodulatory activity of DFAT cells contributes to the healing of alveolar bone defects.

In conclusion, this study demonstrated the efficacy of PLGA scaffolds seeded with DFAT cells in repairing three-wall periodontal defects. The regenerated periodontal tissue restored the original architecture as characterized by the insertion of gingival and principal fibers into the cementum and bone layers. These results indicate that transplanted DFAT cells could promote periodontal tissue regeneration, while PLGA scaffolds prevent gingival invasion by securing a space for restored periodontal tissue, suggesting that the implantation of PLGA/DFAT constructs could be a promising approach in treating periodontal defects.

\section{Acknowledgments}

This work was supported in part by a MEXT KAKENHI (21390528, 15H05037, 15K15724 to MH), and JSPS KAKENHI (16K11703 to TT), MEXT-Supported Program for the Strategic Research Foundation at Private Universities (S1411018) and Nihon University President's Grant for Multidisciplinary Studies.

\section{Conflict of interest}

None declared.

\section{References}

1. Tonetti MS, D'Aiuto F, Nibali L, Donald A, Storry C, Parkar $\mathrm{M}$ et al. (2007) Treatment of periodontitis and endothelial function. N Engl J Med 356, 911-920.

2. Radnai M, Pál A, Novák T, Urbán E, Eller J, Gorzó I (2009) Benefits of periodontal therapy when preterm birth threatens. J Dent Res 88, 280-284.

3. Simpson TC, Needleman I, Wild SH, Moles DR, Mills EJ (2010) Treatment of periodontal disease for glycaemic control in people with diabetes. Cochrane Database Syst Rev 5, CD004714.

4. Taba M Jr, Jin Q, Sugai JV, Giannobile WV (2005) Current concepts in periodontal bioengineering. Orthod Craniofac Res 8, 292-302.

5. Rios HF, Lin Z, Oh B, Park CH, Giannobile WV (2011) Cell- and gene-based therapeutic strategies for periodontal regenerative medicine. J Periodontol 82, 1223-1237.

6. Kawaguchi H, Hirachi A, Hasegawa N, Iwata T, Hamaguchi $\mathrm{H}$, Shiba H et al. (2004) Enhancement of periodontal tissue regeneration by transplantation of bone marrow mesenchymal stem cells. J Periodontol 75, 1281-1287.

7. Hasegawa M, Yamato M, Kikuchi A, Okano T, Ishikawa I (2005) Human periodontal ligament cell sheets can regenerate periodontal ligament tissue in an athymic rat model. Tissue Eng 11, 469-478.

8. Yang Y, Rossi FM, Putnins EE (2010) Periodontal regeneration using engineered bone marrow mesenchymal stromal cells. Biomaterials 31, 8574-8582.

9. Yu N, Oortgiesen DAW, Bronckers ALJJ, Yang F, Walboomers XF, Jansen JA (2013) Enhanced periodontal tissue regeneration by periodontal cell implantation. J Clin Periodontol 40, 698-706.

10. Yamada Y, Ueda M, Hibi H, Baba S (2006) A novel approach to periodontal tissue regeneration with mesenchymal stem cells and platelet-rich plasma using tissue engineering technology: a clinical case report. Int J Periodontics Restorative Dent 26, 363-369.

11. Fraser JK, Wulur I, Alfonso Z, Hedrick MH (2006) Fat tissue: an underappreciated source of stem cells for biotechnology. Trends Biotechnol 24, 150-154.

12. Elabd C, Chiellini C, Massoudi A, Cochet O, Zaragosi LE, Trojani $\mathrm{C}$ et al. (2007) Human adipose tissue-derived multipotent stem cells differentiate in vitro and in vivo into osteocyte-like cells. Biochem Biophys Res Commun 361, 342-348.

13. Akita D, Morokuma M, Saito Y, Yamanaka K, Akiyama Y, Sato $M$ et al. (2014) Periodontal tissue regeneration by transplantation of rat adipose-derived stromal cells in combination with PLGA-based solid scaffolds. Biomed Res 35, 91-103.

14. Akita D, Kano K, Saito-Tamura Y, Mashimo T, SatoShionome M, Tsurumachi N et al. (2016) Use of rat mature adipocyte-derived dedifferentiated fat cells as a cell source for periodontal tissue regeneration. Front Physiol 7, doi: 10.3389/fphys.2016.00050.

15. Matsumoto T, Kano K, Kondo D, Fukuda N, Iribe Y, Tanaka 
$\mathrm{N}$ et al. (2008) Mature adipocyte-derived dedifferentiated fat cells exhibit multilineage potential. J Cell Physiol 215, 210-222.

16. Tsurumachi N, Akita D, Kano K, Matsumoto T, Toriumi T, Kazama T et al. (2016) Small buccal fat pad cells have high osteogenic differentiation potential. Tissue Eng Part C Methods 22, 250-259.

17. Ono H, Oki Y, Bono H, Kano K (2011) Gene expression profiling in multipotent DFAT cells derived from mature adipocytes. Biochem Biophys Res Commun 407, 562-567.

18. Doğan A, Özdemir A, Kubar A, Oygür T (2003) Healing of artificial fenestration defects by seeding of fibroblast-like cells derived from regenerated periodontal ligament in a dog: a preliminary study. Tissue Eng 9, 1189-1196.

19. Zhao M, Jin Q, Berry JE, Nociti FH Jr, Giannobile WV, Somerman MJ (2004) Cementoblast delivery for periodontal tissue engineering. J Periodontol 75, 154-161.

20. Tobita M, Uysal AC, Ogawa R, Hyakusoku H, Mizuno H (2008) Periodontal tissue regeneration with adipose-derived stem cells. Tissue Eng Part A 14, 945-953.

21. Li H, Yan F, Lei L, Li Y, Xiao Y (2009) Application of autologous cryopreserved bone marrow mesenchymal stem cells for periodontal regeneration in dogs. Cells Tissues Organs 190, 94-101.

22. Nuñez J, Sanz-Blasco S, Vignoletti F, Muñoz F, Arzate H, Villalobos C et al. (2012) Periodontal regeneration following implantation of cementum and periodontal ligament-derived cells. J Periodontal Res 47, 33-44.

23. Yamanaka K, Yamamoto K, Sakai Y, Suda Y, Shigemitsu Y, Kaneko T et al. (2015) Seeding of mesenchymal stem cells into inner part of interconnected porous biodegradable scaffold by a new method with a filter paper. Dent Mater J 34, 78-85.

24. Nemcovsky CE, Zahavi S, Moses O, Kebudi E, Artzi Z, Beny L et al. (2006) Effect of enamel matrix protein derivative on healing of surgical supra-infrabony periodontal defects in the rat molar: a histomorphometric study. J Periodontol 77, 996-1002.

25. Oortgiesen DA, Yu N, Bronckers AL, Yang F, Walboomers XF, Jansen JA (2012) A three-dimensional cell culture model to study the mechano-biological behavior in periodontal ligament regeneration. Tissue Eng Part C Methods 18, 81-89.

26. Tsunori K, Sato S, Hasuike A, Manaka S, Shino H, Sato N et al. (2015) Effects of intermittent administration of parathyroid hormone on bone augmentation in rat calvarium. Implant Dent 24, 142-148.

27. Bizenjima T, Seshima F, Ishizuka Y, Takeuchi T, Kinumatsu T, Saito A (2015) Fibroblast growth factor-2 promotes healing of surgically created periodontal defects in rats with early, streptozotocin-induced diabetes via increasing cell proliferation and regulating angiogenesis. J Clin Periodontol $42,62-71$.
28. Pellegrini G, Seol YJ, Gruber R, Giannobile WV (2009) Pre-clinical models for oral and periodontal reconstructive therapies. J Dent Res 88, 1065-1076.

29. King GN, King N, Cruchley AT, Wozney JM, Hughes FJ (1997) Recombinant human bone morphogenetic protein-2 promotes wound healing in rat periodontal fenestration defects. J Dent Res 76, 1460-1470.

30. Tsumanuma Y, Iwata T, Washio K, Yoshida T, Yamada A, Takagi R et al. (2011) Comparison of different tissue-derived stem cell sheets for periodontal regeneration in a canine 1-wall defect model. Biomaterials 32, 5819-5825.

31. Dogan A, Ozdemir A, Kubar A, Oygür T (2002) Assessment of periodontal healing by seeding of fibroblast-like cells derived from regenerated periodontal ligament in artificial furcation defects in a dog: a pilot study. Tissue Eng 8, 273-282.

32. Ogawa K, Miyaji H, Kato A, Kosen Y, Momose T, Yoshida $\mathrm{T}$ et al. (2016) Periodontal tissue engineering by nano betatricalcium phosphate scaffold and fibroblast growth factor-2 in one-wall infrabony defects of dogs. J Periodontal Res 51, 758-767.

33. Cai X, Yang F, Yan X, Yang W, Yu N, Oortgiesen DA et al. (2015) Influence of bone marrow-derived mesenchymal stem cells pre-implantation differentiation approach on periodontal regeneration in vivo. J Clin Periodontol 42, 380-389.

34. Kikuta S, Tanaka N, Kazama T, Kazama M, Kano K, Ryu $\mathrm{J}$ et al. (2013) Osteogenic effects of dedifferentiated fat cell transplantation in rabbit models of bone defect and ovariectomy-induced osteoporosis. Tissue Eng Part A 19, 1792-1802.

35. Chen L, Tredget EE, Wu PY, Wu Y (2008) Paracrine factors of mesenchymal stem cells recruit macrophages and endothelial lineage cells and enhance wound healing. PLoS One 3, e1886.

36. Gnecchi M, Zhang Z, Ni A, Dzau VJ (2008) Paracrine mechanisms in adult stem cell signaling and therapy. Circ Res 103, 1204-1219.

37. Mooney DJ, Vandenburgh H (2008) Cell delivery mechanisms for tissue repair. Cell Stem Cell 2, 205-213.

38. Chen FM, Jin Y (2010) Periodontal tissue engineering and regeneration: current approaches and expanding opportunities. Tissue Eng Part B Rev 16, 219-255.

39. Beertsen W, McCulloch CA, Sodek J (1997) The periodontal ligament: a unique, multifunctional connective tissue. Periodontol 2000 13, 20-40.

40. Bartold PM, Walsh LJ, Narayanan AS (2000) Molecular and cell biology of the gingiva. Periodontol 2000 24, 28-55.

41. Maruyama T, Fukuda N, Matsumoto T, Kano K, Endo M, Kazama M et al. (2015) Systematic implantation of dedifferentiated fat cells ameliorated monoclonal antibody 1-22-3-induced glomerulonephritis by immunosuppression with increases in TNF-stimulated gene 6 . Stem Cell Res Ther 6,80 . 